\title{
Avaliação postural e de deformidades em idosos acamados de uma instituição de longa permanência
}

\author{
Victor Ignácio Freire de Assis*, Alessandra Paiva de Castro Vidal ${ }^{\star \star}$, Fernanda Moura Vargas Dias***
}

\section{Resumo}

A imobilidade produz alterações fisiopatológicas musculoesqueléticas que ocasionam deformidades e alterações posturais. Sem intervenção precoce, as alterações posturais afetam a qualidade de vida do idoso e predispõem ao aparecimento de doenças. Muitos idosos, por não terem famílias, ou condição estável, são institucionalizados. A institucionalização pode contribuir para a inatividade. Dessa forma, o presente estudo tem o objetivo de avaliar o posicionamento no leito e a mobilidade articular em idosos acamados de uma instituição de longa permanência (ILP). Para isso, realizou-se um estudo transversal analítico com quatorze idosos acamados de uma ILP de Vitória, Espírito Santo. Eles foram avaliados por meio de anamnese e exame físico. Foram investigadas deformidades e alterações posturais com um formulário estruturado para inspeção da coluna, membros superiores e inferiores. Os idosos acamados apresentaram idade de $81,1 \pm 12,6$ anos e tempo de institucionalização de 9,6 $2 \pm 6,0$ anos. A maior parte deles $(64,2 \%)$ apresentava alteração postural nos cotovelos, quadris e joelhos, que estavam em flexão. A sensação final de movimento estava normal em todas as articulações na maioria dos idosos. A articulação do ombro apresentou-se com limitação de movimento mais frequentemente $(92,85 \%)$. Além disso, nas articulações de punho, quadril, joelho e tornozelo também existiam limitações. Sabe-se que as limitações do movimento articular podem ocasionar deformidades permanentes. O imobilismo, em idosos acamados residentes em ILP, é um fator causador das alterações do posicionamento e da mobilidade articular. Dessa forma, sugere-se que idosos acamados residentes em ILPs sejam estimulados frequentemente em relação às trocas de decúbito e recebam cuidado especializado para prevenção de deformidades articulares.

Palavras-chave: Idoso. Saúde do idoso institucionalizado. Contratura. Amplitude de movimento. Repouso em cama.

* Aluno de iniciação científica do curso de Fisioterapia da Universidade Federal do Espírito Santo - Ufes. Bolsista Facitec. E-mail: victorfdeassis@gmail.com

** Doutora em Fisioterapia pela Universidade Federal de São Carlos. Professora adjunta do curso de Fisioterapia da Ufes. E-mail: alessandrapaiva2@yahoo.com.br

**** Doutora em Ciências Fisiológicas pela Ufes. Professora adjunta e coordenadora do curso de Fisioterapia da Ufes. Endereço para correspondência: Rua Luiz Fraga, no 70, Maruípe, Vitória, Espírito Santo, CEP 29043-180. E-mail: fernandamvargas@yahoo.com.br/fernanda.dias@ufes.br

$\rightarrow$ http://dx.doi.org/10.5335/rbceh.v12i2.4926

Recebido em: 20/04/2015. Aceito em: 23/10/2015 


\section{Introdução}

O desenvolvimento tecnológico dos últimos anos possibilitou que as pessoas da sociedade moderna tenham maior expectativa de vida. Em conjunto com a queda da natalidade e da mortalidade, a longevidade contribui para o envelhecimento da população. Portanto, o processo de transição demográfica imprime um acelerado aumento do número de idosos em relação aos demais grupos etários (PARAHYBA; SIMÕES, 2006; POLARO et al., 2012).

A insuficiente rede de suporte à família e ao idoso que afeta, geralmente, a camada mais pobre da população, justifica a necessidade da existência de instituições de longa permanência para idosos. Muitos dos idosos institucionalizados não têm famílias, ou se as têm, elas são carentes de recursos materiais, o que impossibilita o convívio e cuidado domiciliar (POLARO et al., 2012).

Embora alguns idosos sejam capazes de realizar suas atividades de vida diária (AVD), quando institucionalizados, eles passam a maior parte do tempo sentados ou deitados. Muitas vezes isso ocorre devido à falta de condições estruturais das instituições de longa permanência para idosos (ILP), como espaço físico insuficiente, instalações sem adaptações e recursos humanos escassos. Soma-se a isso, o medo de o idoso sofrer alguma queda. Além disso, a limitação funcional aumenta com o tempo e há, da parte dos cuidadores, uma subestimação da capacidade do idoso em realizar suas funções. Dessa forma, os residentes permanecem boa parte do tempo deitados ou sentados no leito, o que pode ocasionar graves consequências à saúde, como a osteopo- rose, atrofia muscular, artrites, úlceras por pressão, disfunção cardíaca, dor, constipação, atelectasia, incontinência urinária e fecal e depressão, que são características da síndrome do imobilismo (LAZOWSKI et al., 1999; CHEN, 2010).

Vale ressaltar, ainda, que a restrição ao leito contribui de forma direta para a formação de contraturas (WAGNER; CLEVENGER, 2010; WAGNER et al.; 2008; MOLLINGER; STEFFEN; BROWN, 1993). As contraturas podem ser definidas como um encurtamento adaptativo do músculo com consequente deposição de fibrose tecidual, além de alterações articulares, gerando restrição ao movimento passivo (WAGNER; CLEVENGER, 2010).

Muitas vezes, o cuidado preventivo em relação aos efeitos deletérios do imobilismo, como a formação de contraturas, não faz parte da rotina dos cuidadores nas instituições de longa permanência. Diante disso, o objetivo do presente estudo é avaliar a postura e as deformidades dos idosos acamados de uma ILP em Vitória, Espírito Santo. A compreensão dos possíveis efeitos do imobilismo na postura e mobilidade articular em idosos institucionalizados pode contribuir para que estratégias específicas de prevenção de complicações sejam elaboradas.

\section{Materiais e métodos}

O presente estudo é do tipo observacional transversal analítico, e foi previamente aprovado pelo Comitê de Ética em Pesquisa da Universidade Federal do Espírito Santo, sob o Parecer Consubstanciado $\mathrm{n}^{\circ} 494.084$.

Foi utilizada uma amostra de conveniência, na qual todos os dezoito idosos 
acamados residentes de uma ILP pública de Vitória (Espírito Santo, Brasil) foram convidados a participar do estudo. Como critério de elegibilidade para participar da amostra, o idoso deveria ter idade igual ou maior que 60 anos e ser acamado. Foram excluídos da amostra um idoso que faleceu e um idoso que foi internado no período de coleta do estudo. Além desses, dois idosos não aceitaram participar da pesquisa $\mathrm{e}$ também foram excluídos. Dessa forma, a amostra final utilizada na pesquisa foi de quatorze idosos. Os idosos, ou os seus representantes legais, foram previamente informados sobre o estudo, e aqueles que estavam de acordo assinaram o termo de consentimento livre e esclarecido (TCLE).

No primeiro dia de atendimento, os pesquisadores fizeram a leitura do TCLE para todos os idosos, e aqueles que estavam de acordo em participar da pesquisa o assinaram, em duas vias. Uma via ficou com os pesquisadores e a outra foi entregue ao participante. Após a assinatura, os idosos foram avaliados por meio de uma anamnese para a coleta de dados sociodemográficos. $\mathrm{O}$ exame físico constituiu-se de: a) avaliação do posicionamento do idoso no leito; b) avaliação da sensação final de movimento das principais articulações; c) avaliação do jogo articular; d) avaliação da presença de limitação da amplitude de movimento.

Para avaliação do posicionamento no leito foi utilizado um formulário estruturado para inspeção do posicionamento de cabeça, ombro, cotovelo, punho, quadril, joelho e tornozelo. Durante a inspeção, o avaliador verificou se as articulações apresentavam-se dentro ou fora do padrão anatômico (posicionamento neutro).
Foi realizada a máxima amplitude de movimento passiva do segmento, com o objetivo de avaliar a sensação final do movimento, e que pôde ser classificada em osso com osso, estiramento tecidual e/ou aproximação de partes moles, dependendo da região avaliada. $O$ resultado da avaliação da sensação final de movimento foi categorizado em normal, alterado e não avaliado (HAYER; PERTENSEN, 2001).

Para a avaliação do jogo articular foi realizada a mobilização de cada articulação, classificando a oscilação articular em muito movimento, pouco movimento ou normal (ABBOTT, 2001).

A avaliação da presença de limitação da amplitude de movimento foi realizada por meio do goniômetro. Foi realizada a movimentação passiva do segmento articular avaliado e mensurada a amplitude do movimento em graus. Caso o idoso não executasse 0 arco de movimento completo de acordo com os valores de referência para cada articulação, a limitação da amplitude de movimento era considerada presente (WAGNER et al., 2008).

Os resultados foram analisados descritivamente, a fim de caracterizar a amostra. Os dados foram tabulados em Microsoft Office Excel 2007, e a análise estatística foi realizada no software estatístico Graph Pad Prism 5. Demonstraram-se os resultados em média \pm desvio padrão, frequência absoluta e relativa.

\section{Resultados}

A amostra foi composta por quatorze idosos com média de idade de $81,1 \pm 12,6$ anos e de tempo de permanência de 9,6 $\pm 6,0$ anos. A maior parte era do sexo feminino, com predominância das raças 
branca e parda. A amostra foi composta, principalmente, por viúvos com comunicação preservada, a maioria com ensino fundamental incompleto e analfabeta (Tabela 1).

Tabela 1 - Características dos idosos acamados residentes em instituição de longa permanência

\begin{tabular}{|c|c|}
\hline \multicolumn{2}{|c|}{ Características dos idosos acamados $(n=14)$} \\
\hline Variáveis & Média \pm DP \\
\hline Idade (anos) & $81,1 \pm 12,6$ \\
\hline \multirow[t]{2}{*}{ Tempo de permanência no asilo (anos) } & $9,6 \pm 6,0$ \\
\hline & $\mathrm{Fr} \%(\mathrm{Fa})$ \\
\hline \multicolumn{2}{|l|}{ Sexo } \\
\hline Feminino & $64,29 \%(9)$ \\
\hline Masculino & $35,71 \%(5)$ \\
\hline \multicolumn{2}{|l|}{ Raça } \\
\hline Branca & $42,86 \%(6)$ \\
\hline Negra & $14,28 \%(2)$ \\
\hline Parda & $42,86 \%(6)$ \\
\hline \multicolumn{2}{|l|}{ Estado civil } \\
\hline Casado & $14,28 \%(2)$ \\
\hline Solteiro & $21,43 \%(3)$ \\
\hline Viúvo & $42,86 \%(6)$ \\
\hline Divorciado & $7,15 \%(1)$ \\
\hline Não informado & $14,28 \%(2)$ \\
\hline \multicolumn{2}{|l|}{ Comunicação } \\
\hline Preservada & $64,29 \%(9)$ \\
\hline Não fala & $35,71 \%(5)$ \\
\hline \multicolumn{2}{|l|}{ Escolaridade } \\
\hline Analfabeto & $35,71 \%(5)$ \\
\hline Ensino fundamental incompleto & $42,86 \%(6)$ \\
\hline Ensino superior incompleto & $7,15 \%(1)$ \\
\hline Não informado & $14,28 \%(2)$ \\
\hline \multicolumn{2}{|l|}{ Fonte: dos autores. } \\
\hline \multicolumn{2}{|c|}{$\begin{array}{l}\text { De acordo com a Tabela } 2 \text {, a maior } \\
\text { te dos idosos encontrava-se com a } \\
\text { eça em rotação neutra e à esquerda } \\
\text { forma equivalente. Em relação à in- } \\
\text { ıação, a maioria estava com a cabeça }\end{array}$} \\
\hline
\end{tabular}

em posição neutra, sendo relevante, também, a rotação à esquerda. Os ombros encontravam-se em posição neutra, e os cotovelos em flexão. Os punhos foram encontrados em posição neutra, enquanto os quadris e joelhos apresentavam-se em flexão. A maior parte dos tornozelos observados estava em posição neutra.

Tabela 2 - Avaliação do posicionamento no leito dos idosos acamados

\begin{tabular}{|c|c|c|c|}
\hline \multicolumn{4}{|c|}{$\begin{array}{l}\text { Avaliação do posicionamento no leito dos idosos } \\
\text { acamados }(n=14)\end{array}$} \\
\hline Cabeça & $\mathrm{Fr} \%(\mathrm{Fa})$ & Fr \%(Fa) & $\mathrm{Fr} \%(\mathrm{Fa})$ \\
\hline Rotação & $\begin{array}{l}\text { Neutro } \\
42,86 \%(6)\end{array}$ & $\begin{array}{l}\text { à direita } \\
14,28 \%(2)\end{array}$ & $\begin{array}{l}\text { à esquerda } \\
42,86 \%(6)\end{array}$ \\
\hline Inclinação & $\begin{array}{l}\text { Neutro } \\
42,86 \%(6)\end{array}$ & $\begin{array}{l}\text { à direita } \\
21,43 \%(3)\end{array}$ & $\begin{array}{l}\text { à esquerda } \\
35,71 \%(5)\end{array}$ \\
\hline Ombro & Direito & Esquerdo & \\
\hline Neutro & $92,85 \%(13)$ & $92,85 \%(13)$ & \\
\hline Flexão & $7,15 \%(1)$ & $7,15 \%(1)$ & \\
\hline Extensão & $0,00 \%(0)$ & $0,00 \%(0)$ & \\
\hline Cotovelo & Direito & Esquerdo & \\
\hline Neutro & $35,71 \%(5)$ & $28,57 \%(4)$ & \\
\hline Flexão & $64,29 \%(9)$ & $71,43 \%(10)$ & \\
\hline Extensão & $0,00 \%(0)$ & $0,00 \%(0)$ & \\
\hline Punho & Direito & Esquerdo & \\
\hline Neutro & $85,72 \%(12)$ & $85,72 \%(12)$ & \\
\hline Flexão & $14,28 \%(2)$ & $14,28 \%(2)$ & \\
\hline Extensão & $0,00 \%(0)$ & $0,00 \%(0)$ & \\
\hline Quadril & Direito & Esquerdo & \\
\hline Neutro & $35,71 \%(5)$ & $28,57 \%(4)$ & \\
\hline Flexão & $64,29 \%(9)$ & $71,43 \%(10)$ & \\
\hline Extensão & $0,00 \%(0)$ & $0,00 \%(0)$ & \\
\hline Joelho & Direito & Esquerdo & \\
\hline Neutro & $35,71 \%(5)$ & $21,43 \%(3)$ & \\
\hline Flexão & $64,29 \%(9)$ & $78,57 \%(11)$ & \\
\hline Extensão & $0,00 \%(0)$ & $0,00 \%(0)$ & \\
\hline Tornozelo & Direito & Esquerdo & \\
\hline Dosiflexão & $0,00 \%(0)$ & $0,00 \%(0)$ & \\
\hline Plantiflexão & $35,71 \%(5)$ & $42,86 \%(6)$ & \\
\hline Neutro & $64,29 \%(9)$ & $57,14 \%(8)$ & \\
\hline
\end{tabular}


A Tabela 3 apresenta as caracterís- avaliadas, a sensação final estava dentro ticas da sensação final dos movimentos do esperado, ou seja, normal. articulares. Em todas as articulações

Tabela 3 - Sensação final do movimento articular em idosos acamados residentes em instituição de longa permanência

\begin{tabular}{|c|c|c|c|c|c|c|}
\hline \multirow{3}{*}{ Articulações } & \multicolumn{6}{|c|}{ Sensação final do movimento articular } \\
\hline & \multicolumn{3}{|c|}{ Direito } & \multicolumn{3}{|c|}{ Esquerdo } \\
\hline & Normal & Alterado & NA & Normal & Alterado & NA \\
\hline Ombro & $\mathrm{Fr} \%(\mathrm{Fa})$ & $\mathrm{Fr} \%(\mathrm{Fa})$ & $\mathrm{Fr} \%(\mathrm{Fa})$ & $\mathrm{Fr} \%(\mathrm{Fa})$ & $\mathrm{Fr} \%(\mathrm{Fa})$ & $\mathrm{Fr} \%(\mathrm{Fa})$ \\
\hline Flexão & $92,85 \%(13)$ & $0,00 \%(0)$ & $7,15 \%(1)$ & $85,72 \%(12)$ & $0,00 \%(0)$ & $14,28 \%(2)$ \\
\hline Extensão & $42,86 \%(6)$ & $0,00 \%(0)$ & $57,14 \%(8)$ & $42,86 \%(6)$ & $0,00 \%(0)$ & $57,14 \%(8)$ \\
\hline Abdução & $85,72 \%(12)$ & $0,00 \%(0)$ & $14,28 \%(2)$ & $85,72 \%(12)$ & $0,00 \%(0)$ & $14,28 \%(2)$ \\
\hline Rotação interna & $71,43 \%(10)$ & $0,00 \%(0)$ & $28,57 \%(4)$ & $78,57 \%(11)$ & $0,00 \%(0)$ & $21,43 \%(3)$ \\
\hline Rotação externa & $71,43 \%(10)$ & $0,00 \%(0)$ & $28,57 \%(4)$ & $78,57 \%(11)$ & $0,00 \%(0)$ & $21,43 \%(3)$ \\
\hline \multicolumn{7}{|l|}{ Cotovelo } \\
\hline Flexão & $92,85 \%(13)$ & $0,00 \%(0)$ & $7,15 \%(1)$ & $92,85 \%(13)$ & $0,00 \%(0)$ & $7,15 \%(1)$ \\
\hline Extensão & $64,29 \%(9)$ & $28,57 \%$ (4) & $7,15 \%(1)$ & $57,14 \%(8)$ & $35,71 \%(5)$ & $7,15 \%(1)$ \\
\hline Pronação & $92,85 \%(13)$ & $0,00 \%(0)$ & $7,15 \%(1)$ & $92,85 \%(13)$ & $0,00 \%(0)$ & $7,15 \%(1)$ \\
\hline Supinação & $92,85 \%(13)$ & $0,00 \%(0)$ & $7,15 \%(1)$ & $92,85 \%(13)$ & $0,00 \%(0)$ & $7,15 \%(1)$ \\
\hline \multicolumn{7}{|l|}{ Punho } \\
\hline Flexão & $85,72 \%(12)$ & $0,00 \%(0)$ & $14,28 \%(2)$ & $85,72 \%(12)$ & $0,00 \%(0)$ & $14,28 \%(2)$ \\
\hline Extensão & $85,72 \%(12)$ & $0,00 \%(0)$ & $14,28 \%(2)$ & $85,72 \%(12)$ & $0,00 \%(0)$ & $14,28 \%(2)$ \\
\hline Desvio radial & $78,57 \%(11)$ & $7,15 \%$ (1) & $14,28 \%(2)$ & $78,57 \%(11)$ & $7,15 \%$ (1) & $14,28 \%(2)$ \\
\hline Desvio ulnar & $85,72 \%(12)$ & $0,00 \%(0)$ & $14,28 \%(2)$ & $85,72 \%(12)$ & $0,00 \%(0)$ & $14,28 \%(2)$ \\
\hline \multicolumn{7}{|l|}{ Quadril } \\
\hline Flexão & $57,14 \%(8)$ & $0,00 \%(0)$ & $42,86 \%(6)$ & $57,14 \%(8)$ & $0,00 \%(0$ & $42,86 \%(6)$ \\
\hline Extensão & $14,28 \%(2)$ & $0,00 \%(0)$ & $85,72 \%(12)$ & $14,28 \%(2)$ & $0,00 \%(0$ & $85,72 \%(12)$ \\
\hline \multicolumn{7}{|l|}{ Joelho } \\
\hline Flexão & $64,29 \%$ (9) & $14,28 \%$ (2) & $21,43 \%$ (3) & $92,85 \%(13)$ & $0,00 \%(0)$ & $7,15 \%(1)$ \\
\hline Extensão & $71,43 \%(10)$ & $0,00 \%(0)$ & $28,57 \%(4)$ & $92,85 \%(13)$ & $0,00 \%(0)$ & $7,15 \%(1)$ \\
\hline \multicolumn{7}{|l|}{ Tornozelo } \\
\hline Dorsiflexão & $57,14 \%(8)$ & $0,00 \%(0)$ & $42,86 \%(6)$ & $71,43 \%(10)$ & $0,00 \%(0)$ & $28,57 \%(4)$ \\
\hline Plantiflexão & $57,14 \%(8)$ & $0,00 \%(0)$ & $42,86 \%(6)$ & $71,43 \%(10)$ & $0,00 \%(0)$ & $28,57 \%(4)$ \\
\hline Inversão & $64,29 \%(9)$ & $0,00 \%(0)$ & $35,71 \%(5)$ & $78,57 \%(11)$ & $0,00 \%(0)$ & $21,43 \%$ (3) \\
\hline Eversão & $57,14 \%(8)$ & $0,00 \%(0)$ & $42,86 \%(6)$ & $71,43 \%(10)$ & $0,00 \%(0)$ & $28,57 \%(4)$ \\
\hline
\end{tabular}

Fonte: dos autores.

Nota: não avaliado (NA). 
O jogo articular foi classificado de três formas: normal, muito movimento e pouco movimento. Em relação à articulação do ombro foi possível observar que a maioria dos idosos possuía oscilação normal nessa articulação no lado direito e esquerdo. $\mathrm{Na}$ articulação do cotovelo e na articulação do punho, a oscilação foi normal também em ambos os lados avaliados na maior parte dos idosos. Em relação ao membro inferior, em cerca de $50 \%$ dos idosos as articulações do quadril não foram avaliadas, pois a posição do paciente no leito, as restrições de movimento e/ou a posição da maca no quarto impediram o pesquisador de realizar a avaliação em consonância com a semiotécnica correta. Das articulações do quadril avaliadas, no entanto, a maioria apresentava oscilação normal. A articulação do joelho apresentou-se com oscilação normal em metade dos idosos avaliados. A articulação do tornozelo, em contraste às outras articulações, foi a que apresentou jogo articular mais prejudicado nos idosos acamados (Tabela 4).

Tabela 4 - Jogo articular em idosos acamados residentes em instituição de longa permanência

\begin{tabular}{|c|c|c|c|c|c|c|c|c|}
\hline \multicolumn{9}{|c|}{ Jogo articular em idosos acamados } \\
\hline \multirow[b]{2}{*}{ Articulações } & \multicolumn{4}{|c|}{ Direito } & \multicolumn{4}{|c|}{ Esquerdo } \\
\hline & Normal & \begin{tabular}{|c|} 
Muito \\
movimento
\end{tabular} & $\begin{array}{c}\text { Pouco } \\
\text { movimento }\end{array}$ & NA & Normal & \begin{tabular}{|c|} 
Muito \\
movimento
\end{tabular} & $\begin{array}{c}\text { Pouco } \\
\text { movimento }\end{array}$ & NA \\
\hline & $\mathrm{Fr} \%(\mathrm{Fa})$ & $\mathrm{Fr} \%(\mathrm{Fa})$ & $\mathrm{Fr} \%(\mathrm{Fa})$ & $\mathrm{Fr} \%(\mathrm{Fa})$ & $\mathrm{Fr} \%(\mathrm{Fa})$ & $\mathrm{Fr} \%(\mathrm{Fa})$ & $\mathrm{Fr} \%(\mathrm{Fa})$ & $\mathrm{Fr} \%(\mathrm{Fa})$ \\
\hline Ombro & $57,14 \%(8)$ & $0,00 \%(0)$ & $42,86 \%(6)$ & $0,00 \%(0)$ & $64,29 \%(9)$ & $0,00 \%(0)$ & $35,71 \%(5)$ & $0,00 \%(0)$ \\
\hline Cotovelo & $85,72 \%(12)$ & $0,00 \%(0)$ & $7,14 \%(1)$ & $7,14 \%(1)$ & $78,58 \%(11)$ & $0,00 \%(0)$ & $14,28 \%(2)$ & $7,14 \%(1)$ \\
\hline Punho & $64,29 \%(9)$ & $0,00 \%(0)$ & $21,43 \%(3)$ & $14,28 \%(2)$ & $71,44 \%(10)$ & $0,00 \%(0)$ & $14,28 \%(2)$ & $14,20 \%(2)$ \\
\hline Quadril & $42,86 \%(6)$ & $0,00 \%(0)$ & $7,14 \%(1)$ & $50,0 \%(7)$ & $35,71 \%(5)$ & $7,14 \%(1)$ & $14,28 \%(2)$ & $42,86 \%(6)$ \\
\hline Joelho & $50,0 \%(7)$ & $0,00 \%(0)$ & $28,57 \%(4)$ & $21,43 \%$ (3) & $50,0 \%(7)$ & $7,14 \%$ (1) & $35,71 \%(5)$ & $7,14 \%(1)$ \\
\hline Tornozelo & $14,28 \%$ (2) & $0,00 \%(0)$ & $64,29 \%(9)$ & $21,43 \%$ (3) & $14,28 \%(2)$ & $7,14 \%(1)$ & $64,29 \%(9)$ & $14,28 \%(2)$ \\
\hline
\end{tabular}

Fonte: dos autores.

Nota: não avaliado (NA).

De acordo com a Tabela 5, todas as articulações avaliadas tiveram limitações da amplitude de movimento (ADM). A articulação do ombro direito foi a com maior porcentagem, chegando a $92,85 \%$ das articulações avaliadas. Outras articulações como punho, quadril, joelho e tornozelo também tiveram limitações presentes, sendo os lados direito e esquerdo com valores semelhantes. A articulação em que os idosos apresentaram menor limitação de ADM foi a do cotovelo. 
Tabela 5 - Limitação da amplitude de movimento articular em idosos acamados residentes em instituição de longa permanência

\begin{tabular}{l|r|r|r|r|r|r}
\hline \multicolumn{7}{|c}{ Limitação da amplitude de movimento articular } \\
\hline \multirow{2}{*}{ Articulações } & \multicolumn{5}{|c|}{ Direito } & \multicolumn{1}{c}{ Esquerdo } \\
\cline { 2 - 7 } & \multicolumn{1}{|c|}{ Presente } & \multicolumn{1}{c|}{ Ausente } & NA & Presente & \multicolumn{1}{c}{ Ausente } & NA \\
\hline \multirow{3}{*}{ Ombro } & Fr \%(Fa) & Fr \%(Fa) & Fr \%(Fa) & Fr \%(Fa) & Fr \%(Fa) & Fr \%(Fa) \\
Cotovelo & $92,85 \%(13)$ & $7,15 \%(1)$ & $0,00 \%(0)$ & $71,43 \%(10)$ & $28,57 \%(4)$ & $0,00 \%(0)$ \\
Punho & $35,71 \%(5)$ & $57,14 \%(8)$ & $7,15 \%(1)$ & $42,80 \%(6)$ & $50,00 \%(7)$ & $7,15 \%(1)$ \\
Quadril & $71,43 \%(10)$ & $14,28 \%(2)$ & $14,28 \%(2)$ & $71,43 \%(10)$ & $14,28 \%(2)$ & $14,28 \%(2)$ \\
Joelho & $85,70 \%(12)$ & $0,00 \%(0)$ & $14,28 \%(2)$ & $71,43 \%(10)$ & $14,28 \%(2)$ & $14,28 \%(2)$ \\
Tornozelo & $71,43 \%(10)$ & $7,15 \%(1)$ & $21,43 \%(3)$ & $78,50 \%(11)$ & $14,28 \%(2)$ & $7,15 \%(1)$ \\
\hline Fonte: dos autoryyyyyy & $71,43 \%(10)$ & $14,28 \%(2)$ & $14,28 \%(2)$ & $78,50 \%(11)$ & $21,43 \%(3)$ & $0,00 \%(0)$ \\
\hline
\end{tabular}

Fonte: dos autores.

Nota: não avaliado (NA).

\section{Discussão}

A rápida e radical transição demográfica ocorrida no Brasil fez com que não houvesse tempo para o governo se estruturar (VERAS, 2009). Por isso, a incipiente formação de políticas públicas voltadas à promoção e à prevenção da saúde e de programas que objetivam a independência funcional do idoso culminou na internação precoce dos idosos em instituições de longa permanência (REIS; CEOLIM, 2007; POLARO et al., 2012).

Em estudos realizados por Fox et al. (2000), Chen (2010) e Wagner et al. (2007), em instituições de longa permanência para idosos, a média de idade das amostras foi próxima a do presente estudo, sugerindo que nessas instituições são prevalentemente encontradas pessoas com média de 80 anos de idade. Além disso, a maioria da amostra foi composta de indivíduos do sexo feminino, corrobo- rando com o presente trabalho. Embora no estudo de Wagner (2007) a maior parte da amostra tenha sido composta por pessoas brancas, em nossa pesquisa houve uma equivalência entre indivíduos brancos e pardos, além de uma minoria negra. No estudo de Chen (2010), um dos critérios de exclusão foi a falta de comunicação, pois para os critérios de elegibilidade daquela pesquisa, todos os indivíduos deveriam estar aptos a se comunicar para que pudessem responder às perguntas dos examinadores. Embora a minoria da amostra deste estudo não estivesse apta a se comunicar, isso não foi considerado um critério de exclusão.

A restrição de movimentos articulares está relacionada ao posicionamento no leito mantido durante longos períodos, contribuindo para o encurtamento adaptativo do músculo, resultando em limitação da amplitude de movimento (FOX et. al., 2000). A mudança regular do posicionamento no leito contribui 
para evitar a formação de úlceras por pressão (JAUL, 2010), entretanto, não é efetiva quanto à prevenção de formação de contraturas, pois os posicionamentos adotados (decúbito dorsal, decúbito lateral direito e esquerdo) mantêm o posicionamento articular em flexão, o que contribui para o encurtamento adaptativo dos músculos. Nesse caso, o ideal é a postura prona, não adotada por nenhum deles, nem por alguns minutos, o que reforça os achados neste estudo.

A sensação final do movimento depende do tipo de movimento (flexão, extensão, por exemplo) e da articulação que está sendo avaliada. Em cada articulação, associada a um tipo de movimento, espera-se encontrar uma sensação final que pode ser classificada em osso com osso, estiramento tecidual ou aproximação de partes moles. Caso a sensação final não seja a esperada, a articulação possui e/ou sofre influência do encurtamento adaptativo do músculo e/ou alterações de cápsula articular, por exemplo, culminando em uma alteração na amplitude de movimento (USHUBA et al., 2006). Neste estudo, para melhor exposição dos dados, a sensação final foi classificada em normal ou alterada. A alteração na sensação final na extensão do cotovelo esperada seria o contato osso com osso. Porém, houve uma restrição à extensão completa deste por estiramento tecidual, devido ao encurtamento causado pela posição fletida durante longo período no leito.

Vale ressaltar ainda que o estiramento tecidual pode ser encontrado no final de uma amplitude de movimento completa ou incompleta. Portanto, somente a sensação final não pode ser considerada um preditor de alteração muscular (encurtamento) ou articular, devendo ser relacionada à amplitude de movimento quando a sensação final de estiramento tecidual já for a esperada. Porém, não foram encontrados artigos na literatura científica que relacionassem a sensação final e a amplitude de movimento.

O jogo articular foi avaliado com o objetivo de caracterizar a origem da restrição do movimento, ou seja, identificar se a contratura era de origem articular ou muscular. Entretanto, na literatura científica, o termo contratura é muito discutido e não há um conceito exato. Sabe-se que com o imobilismo há alterações musculares e articulares. Alguns estudos trazem a relevância da contratura artrogênica, e outros da miogênica, além daqueles que reforçam a falta de importância clínica relevante dessa diferenciação (TRUDEL; UHTHOFF, 2000). No presente estudo, houve alteração na oscilação de várias articulações, mostrando que ocorreram mudanças, não só musculares, mas também articulares na amostra. Isso pode ser explicado pelo tempo de restrição ao leito com ausência de estímulo e/ou mudanças de decúbito, o que atenuaria os efeitos deletérios do imobilismo no âmbito das ILPs (HARBURN; POTTER, 1993).

O estudo realizado por Trudel e Uhthoff (2000) sugere que nos primeiros noventa dias de imobilidade a restrição é principalmente muscular, após esse tempo, a restrição é articular, devido a alterações capsulares. Porém, a maioria das articulações avaliadas neste estudo teve suas oscilações normais, divergindo 
do estudo de Trudel e Uhthoff (2000). Isso pode ser explicado pelo fato de que naquele estudo a articulação foi completamente imobilizada, não sendo permitida qualquer movimentação, enquanto no caso dos idosos restritos ao leito, há a manutenção da articulação, mesmo que com poucos movimentos e de baixa amplitude.

Como discutido, a restrição ao leito gera um encurtamento adaptativo do músculo e consequentemente a limitação da ADM passiva. De acordo com Trudel e Uhthoff (2000), Fox et al. (2000) e Wagner (2007), essas características são sugestivas de contraturas. O termo contratura pode ser definido como uma restrição ao movimento passivo, resultante de fibrose tecidual.

De acordo com Resnick (2000), que avaliou a presença de contraturas em 59 residentes de ILP, ficou evidenciado que $32 \%$ dos indivíduos avaliados tinham contraturas em membros superiores, e $26 \%$ nos membros inferiores. Ainda, no estudo de Wagner (2007), no qual foi analisada a presença de contraturas em idosos acamados e institucionalizados, identificou-se que $45 \%$ deles apresentavam múltiplas contraturas. Neste estudo, em relação aos membros superiores, o ombro foi o mais afetado nos sujeitos da pesquisa.

Diante disso, cabe ao fisioterapeuta um papel importante na prevenção e no tratamento das contraturas, mantendo a amplitude de movimento funcional, a mobilidade no leito e a realização de AVD, e auxiliando a proporcionar maior qualidade de vida e independência aos idosos, reduzindo gastos públicos com as consequências do imobilismo no leito.

\section{Conclusões}

Os idosos acamados institucionalizados integrantes desta pesquisa apresentaram predominantemente a postura no leito com rotação de cabeça, cotovelos, quadris e joelhos em flexão. O ombro direito foi a articulação com maior grau de limitação da amplitude de movimento, embora punhos, quadris, joelhos e tornozelos também tivessem com limitações presentes. O jogo articular e a sensação final de movimento apresentaram-se normais na avaliação.

Conclui-se que o imobilismo em idosos acamados residentes em ILP afeta, de forma direta, o posicionamento e a mobilidade articular, ocasionando limitações na $\mathrm{ADM}$ que podem gerar deformidades articulares permanentes. Isso impede a realização das AVDs pelos idosos, tornando-os mais dependentes de cuidadores para suas necessidades básicas. Dessa forma, sugere-se que idosos acamados residentes em ILP sejam estimulados frequentemente em relação às trocas de decúbito, mas também recebam cuidado especializado contínuo para prevenção de deformidades articulares.

É importante ressaltar como limitações da pesquisa que os idosos apresentam disfunções sistêmicas, com causa, história e evolução diferentes, o que gera múltiplos comprometimentos osteomioarticulares e, consequentemente, uma grande dificuldade de generalização dos resultados encontrados. Além disso, salienta-se que a avaliação física em acamados torna-se, muitas vezes, difícil de ser realizada pelas restrições impostas à movimentação pelo próprio decúbito, necessitando de métodos mais específicos e objetivos. 


\section{Evaluation of the positioning in bed and joint mobility in bedridden elderly long term care institutions}

\section{Abstract}

Immobility produces musculoskeletal pathophysiological changes that cause deformities and postural changes. Without early intervention, postural changes affect the quality of life of the elderly and predispose diseases. Many seniors, for not having families, or stable condition, are institutionalized. The institutionalization can contribute to inactivity. Thus, this study aimed to evaluate of the positioning in bed and joint mobility in bedridden elderly long-term care institutions (LSI). For this, there was an analytical cross-sectional study in 14 elderly living in ILP in Vitória, Espírito Santo. They were evaluated by history and physical examination. Deformities and postural changes by a form structured to inspection column, upper and lower limbs were investigated. The immobility showed age of $81.1 \pm 12.6$ years and time of institutionalization of $9.6 \pm 6.0$ years. Most of them $(64.2 \%)$ had postural change in the elbows, hips and knees, which were in flexion. The final movement feeling was normal in all joints in most elderly. The shoulder joint presented with limitation of movement more frequently $(92.85 \%)$. In addition, the joints of the wrist, hip, knee and ankle there were also limitations. It is known that the limitations of motion can cause permanent deformity. Standing still, in elderly residents in ILP, this is a causative factor of changes in the positioning and joint mobility. Thus, it is suggested that elderly residents in ILP were often encouraged in relation to decubitus exchanges and receive specialized care to prevent joint deformities.

Keywords: Elderly. Health of institutionalized elderly. Contracture. Range of motion. Bed rest.

\section{Referências}

ABBOTT, J. H. Mobilization with movement applied to the elbow affects shoulder range of movement in subjects with lateral epicondylalgia. Manual Therapy, v. 6, n. 3, p. 170-177, 2001.

CHEN, Y. M. Perceived barriers to physical activity among older adults residing in longterm care institutions. Journal of Clinical Nursing, v. 19, p. 432-439, 2010.

FOX, P. et al. Effectiveness of a bed positioning program for treating older Adults with knee contractures who are institutionalized. Journal of the Americam Physical Therapy Association, n. 80, p. 363-372, 2000.

HAYES, K. W.; PETERSEN, C. M. Reliability of Assessing End-feel and Pain and Resistance Sequence in Subjects with Painful Shoulders and Knees. J Orthop Sports Phys Ther, v. 31, n. 8, p. 432-445, 2001.

HARBURN, K. L.; POTTER, P. J. Spasticity and contractures. Phys Med Rehabil State of the Art Ver, n. 7, p. 113-132, 1993.

JAUL, E. Assessment and Management of Pressure Ulcers in the Elderly Current Strategies. Drugs \& Aging, v. 27, n. 4, p. 311-325, 2010.

LAZOWSKI, D. A. et al. A randomized outcome evaluation of group exercise programs in long-term care institutions. Journal of Gerontology: Medical Sciences, Oxford, a. 54, n. 12, p. 621-662, 1999.

MOLLINGER, L. A.; STEFFEN, T. M.; BROWN, M. Knee flexion contractures in institutionalized elderly: prevalence, severity, stability, and related variables. Physical Therapy, n. 73, p. 437-446, 1993.

POLORO, S. H. I. et al. Idosos residentes em instituições de longa permanência para idosos da região metropolitana de Belém-PA. $R e$ vista Brasileira de Geriatria e Gerontologia, Rio de Janeiro, v. 15, n. 4, p. 777-784, 2012. 
PARAHYBA, I.; SIMÕES, C. C. S. A prevalência de incapacidade funcional em idosos no Brasil. Ciência \& Saúde Coletiva, Rio de Janeiro, v. 11, n. 4, p. 967-974, 2006.

REIS, P. O.; CEOLIM, M. F. O significado atribuído ao ser idoso por trabalhadores de instituições de longa permanência. Revista da Escola de Enfermagem da USP, São Paulo, v. 41, n. 1, p. 57-64, 2007.

RESNICK B. Functional performance and exercise of older adults in long term care settings. Journal of Gerontological Nursing, n. 26, p. 7-16, 2000.

TRUDEL, G.; UHTHOFF, H. K. Contractures secondary to immobility: is the restriction articular or muscular? An experimental longitudinal study in the rat knee. Archives of Physical Medicine and Rehabilitation, v. 81 , n. 1, p. 6-13, 2000.

USHUBA, M. et al. Effect of heat in increasing the range of knee motion after the development of a joint contracture: an experiment with animal model. Archives of Physical Medicine and Rehabilitation, n. 87, p. 247-253, 2006.

VERAS, R. Envelhecimento populacional contemporâneo: demandas, desafios e inovações. Revista de Saúde Pública, São Paulo, v. 43, n. 3, p. 548-554, 2009.

WAGNER, L. M.; CLEVERNGER, C. Contractures in nursing home residents. Geriatric Nursing, v. 29, n. 4, p. 259-266, 2010.

WAGNER, L. M. et al. Contractures in frail nursing home residents. Geriatric Nursing, v. 29, n. 4, p. 259-266, 2008. 